\title{
Tratamento de insuficiência cardíaca com benazepril em cães com cardiomiopatia dilatada e endocardiose
}

[Treatment of congestive heart failure with benazepril in dogs with dilated cardiomyopathy and endocardiosis]

\author{
P.M. Pereira ${ }^{1 *}$, A.A. Camacho ${ }^{2}$, H.A. Morais $^{3}$ \\ ${ }^{1}$ Departamento de Clínicas Veterinárias - Centro de Ciências Agrárias \\ Universidade Estadual de Londrina - Londrina, PR \\ ${ }^{2}$ Faculdade de Ciências Agrárias e Veterinárias - Universidade Estadual Paulista - Jaboticabal, SP \\ ${ }^{3}$ Department of Medical Sciences - University of Wisconsin - Madison, WI
}

\begin{abstract}
RESUMO
Foram avaliados os efeitos clínicos do benazepril, um inibidor da enzima de conversão da angiotensina de ação prolongada, em cães com insuficiência cardíaca congestiva (ICC) secundária à endocardiose de mitral ou cardiomiopatia dilatada. O medicamento foi administrado na dose de 0,25 a $0,5 \mathrm{mg} / \mathrm{kg} / \mathrm{dia}$. Diuréticos, digitálicos e fármacos antiarrítmicos foram usados de acordo com a necessidade de cada paciente. Exames físico, radiográfico e eletrocardiográfico foram realizados nos dias $0,7,28$ e 56 . A gasometria arterial e a bioquímica sérica foram avaliadas nos dias 0 e 56 . Os sinais de dispnéia e o estado geral dos pacientes melhoraram em todos os cães após o início do tratamento. Houve diminuição na freqüência da tosse e não houve alterações no eletrocardiograma, exceto pela diminuição na amplitude e na duração da onda P. Nenhum efeito colateral foi observado. Conclui-se que o benazepril é um inibidor da enzima de conversão da angiotensina, eficaz e bem tolerado no tratamento da ICC no cão.
\end{abstract}

Palavras-chave: cão, angiotensina, inibidor, benazepril, insuficiência cardíaca

\begin{abstract}
Clinical effects of benazepril, a long acting angiotensin-converting enzyme (ACEi), in dogs with naturally-occurring congestive heart failure (CHF) caused by mitral endocardiosis or dilated cardiomyopathy were studied. The drug was given orally at a dose of 0.25 to $0.5 \mathrm{mg} / \mathrm{kg} /$ day. Diuretics, digitalics, and antiarrhtyhmic drugs were given as needed. Physical, radiographic, and eletrocardiographic examination were performed at days $0,7,28$, and 56. Serum biochemistry and arterial blood gases were obtained at days 0 and 56. Signs of dyspnea and general condition improved in all dogs. Cough decreased in frequency. The electrocardiogram did not change with benazepril use except for a decrease in $P$ wave amplitude and duration. No adverse effects related to the use of benazepril were observed. Benazepril is an effective and well tolerated ACEi for the treatment of CHF in dogs.
\end{abstract}

Keywords: dog, angiotensin, inhibitor, benazepril, heart failure

Recebido para publicação em 27 de fevereiro de 2004

Recebido para publicação, após modificações, em 29 de setembro de 2004

Endereço para correspondência (corresponding address)

Av. Pedro Marques, 221 - Jd. Universitário

14872-222 - Jaboticabal, SP

E-mail: patmendes@netsite.com.br 


\section{INTRODUÇÃO}

A insuficiência cardíaca congestiva (ICC) é uma síndrome clínica, causada em cães principalmente por alterações miocárdicas ou valvares (Roth, 1993; Schlesinger e Rubin, 1994; Buchanan, 1999). A diminuição crônica do débito cardíaco leva à diminuição da pressão arterial, ativando mecanismos compensatórios, como o sistema renina-angiotensina (SRA) e o sistema nervoso simpático, que tentam restabelecê-la (Snyder, 1991; Knight,1992). Esses mecanismos causam primariamente taquicardia, vasoconstrição periférica e retenção de sódio e água (Awan e Mason, 1996; Camacho, 1996), elevando a pressão arterial e o débito cardíaco, o que favorece a perfusão tissular (Awan e Mason, 1996; Camacho, 1996). Os mecanismos compensatórios, ao agirem cronicamente, contribuem para a evolução dos sinais clínicos observados nos pacientes com insuficiência cardíaca. A retenção de sódio e água leva à congestão, ascite, efusão pleural e edema pulmonar e dos membros. Outros sinais incluem cansaço, intolerância ao exercício, dispnéia, taquicardia, presença de ritmo de galope, sopros e arritmias (Calvert, 1991; Roth, 1993; Camacho, 1996; De Morais, 2000).

Os mecanismos compensatórios ativados durante a ICC são os principais responsáveis pela progressão da doença primária (Calvert, 1991; Roth, 1993; De Morais, 2000). Vasoconstrição, taquicardia e aumento da contratilidade intensificam o consumo de oxigênio pelo miocárdio (MVO2), diminuindo a vida útil de suas células. A ativação neuroendócrina (sistema renina-angiotensina e noradrenalina) interfere na síntese protéica intracelular, causando hipertrofia e morte celular, o que aumenta a sobrecarga de trabalho, acelerando a morte das células remanescentes (De Morais, 2000). Isso gera um círculo vicioso, com agravamento progressivo e constante da doença cardíaca até a morte do paciente (De Morais, 2000). A angiotensina II é um vasoconstritor potente que estimula a liberação de aldosterona (reabsorção de sódio e água) e ADH (hormônio antidiurético), aumenta o tono simpático, aumenta a sede, estimula direta e indiretamente o remodelamento vascular através da diminuição da síntese de óxido nítrico e inativação da bradicinina pela enzima de conversão da angiotensina (ECA) (Weber, 1995; De Morais, 2000), levando à hiperplasia, à hipertrofia e à apoptose das células musculares lisas dos vasos (Katz, 1994; Ponikowski et al., 1998).

O bloqueio farmacológico desses hormônios ou de suas vias metabólicas prolonga a sobrevida de pacientes com ICC (Katz, 1994). O tempo de sobrevivência em cães com ICC devido à cardiomiopatia dilatada (CMD) aumentou após a introdução do inibidor da ECA (enalapril) (Controlled..., 1995; Ettinger et al., 1998). Essa droga diminui a vasoconstrição periférica causada pela angiotensina II e inibe a reabsorção de sódio e água estimulada pela produção e liberação de aldosterona (Insel et al., 1989; Sisson, 1991; Roth, 1993). Outros inibidores da ECA apresentam perfil farmacológico similar e podem ser usados no tratamento da ICC em cães (Hamlin e Nakayama, 1998). O benazepril tem ação por 24 horas, podendo ser administrado uma vez ao dia (King et al., 1995). Ele é excretado tanto por via renal quanto hepática, não necessitando de ajuste na dose em pacientes com insuficiência renal (Kitagawa et al., 2000).

O objetivo deste trabalho foi avaliar o uso clínico do benazepril em cães com ICC moderada a severa, analisando as características físicas, bioquímicas séricas, radiográficas e eletrocardiográficas e os valores da pressão arterial, antes e após o uso do benazepril em cães com ICC naturalmente adquirida.

\section{MATERIAL E MÉTODOS}

Realizou-se um estudo prospectivo utilizando cães com cardiopatias naturalmente adquiridas. Foram utilizados 20 cães, 11 machos e nove fêmeas com idade que variava de seis meses a 14 anos (idade média de oito anos), atendidos pelo Serviço de Cardiologia do Hospital Veterinário da UNESP - Campus de Jaboticabal, dos quais oito cães eram sem raça definida, quatro Terrier Brasileiro, dois Boxer, dois Rottweiler, um Setter Irlandês, um Poodle Miniatura, um Fila Brasileiro e um Yorkshire Terrier. Os animais apresentavam sinais clínicos de ICC classe funcional II (ICC moderada) ou III (ICC severa), sendo 11 cães com cardiomiopatia dilatada e nove com endocardiose valvar. O diagnóstico foi feito mediante rigoroso exame clínico, eletrocardiográfico e radiográfico. Foram excluídos cães com disfunção gastrintestinal 
severa que poderia interferir na absorção do fármaco testado.

O estudo teve duração de 56 dias, com quatro consultas nesse tempo. As consultas foram realizadas na primeira visita e com sete, 28 e 56 dias após o início do tratamento. Foram feitos exames físicos, eletrocardiográficos, radiográficos e aferições da pressão arterial (PA) em todas as visitas. Dosagens séricas de alanina aminotransferase (ALT), fosfatase alcalina (FA), uréia, creatinina, sódio e potássio foram realizadas durante a primeira visita e 56 dias após o início do tratamento. Foram obtidos dados referentes às condições gerais do animal, ao grau de tolerância ao exercício e à resposta ao tratamento empregado. Os exames físicos e a aplicação dos questionários foram realizados pela mesma pessoa em todas as quatro consultas.

Os animais foram tratados para ICC. De acordo com a necessidade, foram administrados diuréticos (furosemida), agentes inotrópicos positivos (digoxina, metil-digoxina) e fármacos antiarrítmicos (diltiazem e propranolol). A dieta não foi modificada. $\mathrm{O}$ único fármaco utilizado por seus efeitos vasodilatadores e de bloqueio da enzima de conversão da angiotensina foi $o$ benazepril. Todos os cães receberam comprimidos de benazepril ${ }^{1}$, na dose que variou entre 0,25 a $0,5 \mathrm{mg} / \mathrm{kg}$, uma vez ao dia, por via oral, conforme o tamanho do animal.

O eletrocardiograma foi realizado com eletrocardiógrafo ${ }^{2}$ de seis canais, ajustado na velocidade de $50 \mathrm{~mm} / \mathrm{s}$, calibrado para um milivolte igual a um centímetro. Analisaram-se freqüência cardíaca, ritmo, onda $\mathrm{P}$, intervalos $\mathrm{P}$ R, Q-T e S-T, complexo QRS e onda T, sendo que as medidas das ondas e dos segmentos foram realizadas pela derivação II. Os exames radiográficos foram feitos com o aparelho radiográfico $^{3}$ de $800 \mathrm{~mA}$ com $90 \mathrm{kv}$. As radiografias foram obtidas nas posições láterolateral direita e dorsoventral. Foram avaliados a presença radiográfica de cardiomegalia, a congestão pulmonar (aumento de veias pulmonares) e o edema pulmonar cardiogênico (Lord e Suter, 1999). A pressão arterial foi aferida três vezes em cada consulta, utilizando-se

${ }^{1}$ Fortekor -Novartis - São Paulo

${ }^{2}$ RFT - 6 NECK 4

${ }^{3}$ Tridoros $812 \mathrm{E}$ o método oscilométrico ${ }^{4}$. Os valores utilizados foram as médias das aferições de cada consulta, mas os valores discrepantes eram descartados, e a pressão arterial aferida novamente. As concentrações séricas de uréia, creatinina, ALT e FA foram determinadas por meio de espectrofotometria ${ }^{5}$, na faixa visível para as respectivas enzimas, utilizando-se kits comerciais $^{6}$. As concentrações séricas de sódio e potássio foram verificadas por meio de fotômetro de chama ${ }^{7}$.

Os critérios para a determinação da eficácia terapêutica do tratamento foram a diminuição da freqüência e a intensidade da dispnéia e da tosse, a melhora do quadro clínico inicial, a melhora radiográfica da congestão e do edema pulmonar e a ausência de efeitos adversos, como emese, diarréia, hipotensão, depressão, anorexia e azotemia.

Foram comparadas as modificações ocorridas entre as quatro consultas, utilizando-se análise de variância com medidas repetitivas em um fator (tempo). Com as variáveis quantitativas de distribuição normal, procedeu-se à análise de variância com medidas repetitivas e a comparação das médias aritméticas pelo teste Tukey, sempre que $\alpha$ indicasse $\mathrm{P}<0,05$. As variáveis qualitativas e os dados não paramétricos foram avaliados pelos métodos de Friedman e $\chi^{2}$, usando-se o teste StudentNewman-Keuls como pós-teste. $\mathrm{O}$ valor de $\mathrm{P}<0,05$ foi considerado significativo.

\section{RESULTADOS}

Dos 20 cães utilizados, apenas 15 permaneceram vivos até o final do estudo. Quatro animais (três com CMD e um com endocardiose de mitral) que apresentaram ICC classe funcional III morreram por complicações da ICC, e um animal morreu em decorrência de fatores não relacionados ao estudo (carcinoma metastático). Do total de animais tratados e que permaneceram vivos até se completar o estudo, 12 receberam outras medicações além do benazepril (diuréticos,

${ }^{4}$ DIXTAL 2710

${ }^{5}$ Celm E - 225D

${ }^{6}$ Labtest - Ribeirão Preto

${ }^{7}$ Celm FC - 180 
inotrópicos positivos e/ou antiarrítmicos) na primeira consulta.

Ao exame físico inicial, um animal apresentou diminuição da ingestão de água (segundo relato do proprietário) e seis estavam com peso abaixo do esperado. Onze cães apresentavam dificuldades respiratórias durante exercício e/ou repouso, e nove apresentavam tosse, segundo relato dos respectivos proprietários na consulta inicial. Houve diminuição significativa $(\mathrm{P}<0,05)$ no número de animais que apresentaram dispnéia, freqüência da dispnéia durante o dia e severidade da dispnéia a partir de sete dias após início do tratamento (Tab. 1). Não houve alteração no número de animais que apresentaram tosse, porém sua freqüência diminuiu (Tab. 2). À auscultação pulmonar durante a primeira consulta, sete animais apresentavam crepitações, localizadas principalmente nos lobos apicais e diafragmáticos. Durante o experimento, esse número foi diminuindo gradativamente e, ao término do estudo, apenas três animais apresentaram crepitações, mas a diferença não foi significativa $(\mathrm{P}>0,05)$. Dos 15 cães que permaneceram vivos até o fim do estudo, três apresentavam síncope e 12, intolerância ao exercício. Nenhum episódio de síncope foi observado durante o período de tratamento. O número de cães com intolerância ao exercício diminuiu para nove a partir da primeira reavaliação, mas essa diferença não foi significativa. Apenas quatro cães apresentavam mucosas de coloração normal durante a primeira consulta. As mucosas estavam congestas em seis cães, pálidas em três e cianóticas em dois animais. Houve melhora progressiva na coloração das mucosas, e, na última reavaliação, 13 cães apresentaram mucosas normais e dois, mucosas congestas (Tab. 3). Nenhum efeito adverso foi observado durante todo o decorrer do estudo.

Tabela 1. Caracterização da freqüência, do tempo de ocorrência e da intensidade da dispnéia em cães com insuficiência cardíaca congestiva, tratados com benazepril, na dose de 0,25 a $0,5 \mathrm{mg} / \mathrm{kg}$, uma vez ao dia, por via oral, durante 56 dias

\begin{tabular}{lcccc}
\hline & $\begin{array}{c}\text { Consulta } \\
\text { inicial }\end{array}$ & $\begin{array}{c}\text { Reavaliação } \\
7 \text { dias }\end{array}$ & $\begin{array}{c}\text { Reavaliação } \\
28 \text { dias }\end{array}$ & $\begin{array}{c}\text { Reavaliação } \\
56 \text { dias }\end{array}$ \\
\hline Freqüência & $\mathrm{a}$ & $\mathrm{b}$ & $\mathrm{b}$ & $\mathrm{b}$ \\
Dispnéia ausente & 4 & 9 & 11 & 2 \\
Dispnéia rara & 2 & 5 & 4 & 1 \\
Dispnéia freqüente & 8 & 1 & 0 & 0 \\
Dispnéia muito freqüente & 1 & 0 & 0 & $\mathrm{~b}$ \\
Tempo de ocorrência & $\mathrm{a}$ & $\mathrm{b}$ & $\mathrm{b}$ & 12 \\
Dispnéia ausente & 4 & 9 & 11 & 3 \\
Dispnéia durante exercício & 8 & 5 & 4 & 0 \\
Dispnéia em repouso & 3 & 1 & 0 & $\mathrm{~b}$ \\
Intensidade & $\mathrm{a}$ & $\mathrm{b}$ & $\mathrm{b}$ & 2 \\
Dispnéia ausente & 4 & 9 & 11 & 1 \\
Dispnéia leve & 1 & 3 & 2 & 0 \\
Dispnéia moderada & 4 & 1 & 0 & 0 \\
Dispnéia intensa & 5 & 2 & 0 & 2 \\
Dispnéia muito intensa & 1 & 0 & 0 & 2 \\
\hline
\end{tabular}

Letras minúsculas distintas na linha representam diferenças $(\mathrm{P}<0,05)$ entre as médias nas várias consultas.

Tabela 2. Caracterização da freqüência da tosse em cães com insuficiência cardíaca congestiva, tratados com benazepril, na dose de 0,25 a $0,5 \mathrm{mg} / \mathrm{kg}$, uma vez ao dia, por via oral, durante 56 dias

\begin{tabular}{lcccc}
\hline & Consulta inicial & $\begin{array}{c}\text { Reavaliação } \\
7 \text { dias }\end{array}$ & $\begin{array}{c}\text { Reavaliação } \\
28 \text { dias }\end{array}$ & $\begin{array}{c}\text { Reavaliação } \\
56 \text { dias }\end{array}$ \\
\hline Tosse & $\mathrm{a}$ & $\mathrm{b}$ & $\mathrm{ab}$ & $\mathrm{ab}$ \\
Tosse ausente & 6 & 8 & 7 & 7 \\
Tosse rara & 1 & 6 & 7 & 6 \\
Tosse freqüente & 7 & 1 & 1 & 2 \\
Tosse contínua & 1 & 0 & 0 & 0 \\
\hline
\end{tabular}

Letras minúsculas distintas na linha representam diferenças $(\mathrm{P}<0,05)$ entre as médias nas várias consultas. 
Tabela 3. Caracterização da coloração da mucosa oral em cães com insuficiência cardíaca congestiva, tratados com benazepril, na dose de 0,25 a $0,5 \mathrm{mg} / \mathrm{kg}$, uma vez ao dia, por via oral, durante 56 dias

\begin{tabular}{lcccc}
\hline & Consulta inicial & $\begin{array}{c}\text { Reavaliação } \\
7 \text { dias }\end{array}$ & $\begin{array}{c}\text { Reavaliação } \\
28 \text { dias }\end{array}$ & $\begin{array}{c}\text { Reavaliação } \\
56 \text { dias }\end{array}$ \\
\hline Mucosa oral & $\mathrm{a}$ & $\mathrm{b}$ & $\mathrm{b}$ & $\mathrm{b}$ \\
\hline Mucosa normal & 4 & 7 & 12 & 13 \\
Mucosa pálida & 3 & 2 & 2 & 0 \\
Mucosa congesta & 6 & 4 & 1 & 2 \\
Mucosa cianótica & 2 & 2 & 0 & 0 \\
\hline
\end{tabular}

Letras minúsculas distintas na linha representam diferenças $(\mathrm{P}<0,05)$ entre as médias nas várias consultas.

Não houve alteração significativa no tamanho da silhueta cardíaca ao exame radiográfico. Houve resolução radiográfica do edema pulmonar em todos os cães com ICC severa. Após o início do tratamento, o diâmetro das veias pulmonares retornou para os valores normais (menor que a largura da nona costela) em três de seis cães com ICC moderada em que elas se encontravam aumentadas. Ao exame eletrocardiográfico, não houve diminuição da freqüência cardíaca nem alterações no ritmo cardíaco. Taquicardia ventricular ocorreu em apenas um cão na última reavaliação. Houve diminuição significativa da duração da onda $\mathrm{P}(\mathrm{P}<0,05)$ da avaliação inicial $(0,046 \pm 0,008 \mathrm{seg})$ para a reavaliação aos 28 dias $(0,041 \pm 0,003 \mathrm{seg})$. A amplitude da onda $\mathrm{P}$ foi menor durante a reavaliação aos 28 $(0,18 \pm 0,055 \mathrm{mV})$ e $56(0,19 \pm 0,049 \mathrm{mV})$ dias do que na apresentação inicial $(0,23 \pm 0,075 \mathrm{mV})$ e aos sete dias $(0,22 \pm 0,072 \mathrm{mV})$. Os demais intervalos e ondas não variaram. Ocorreu redução das pressões arteriais média, sistólica e diastólica durante a segunda consulta, mas a diferença não foi significativa. Não houve desenvolvimento de hipotensão arterial.

Não houve variação nas concentrações séricas de uréia (de 40,2 $\pm 19,2 \mathrm{mg} / \mathrm{dl}$ para $45,8 \pm 23,8 \mathrm{mg} / \mathrm{dl}$, 14 cães), creatinina (de $1,02 \pm 0,32 \mathrm{mg} / \mathrm{dl}$ para $0,92 \pm 0,29 \mathrm{mg} / \mathrm{dl}, \quad 13$ cães), potássio (de $4,6 \pm 0,58 \mathrm{mmol} / 1$ para $4,7 \pm 0,58 \mathrm{mmol} / 1,13$ cães), alanina aminotransferase (de 29,2 $\pm 38,5 \mathrm{U} / \mathrm{ml}$ para $42,9 \pm 29,7 \mathrm{U} / \mathrm{ml}, 14$ cães) e fosfatase alcalina (de $60,5 \pm 84,4$ UI para $52,3 \pm 68,9 \mathrm{mg} / \mathrm{dl}, 14$ cães). A concentração de sódio aumentou significativamente de $152,1 \pm 6,7 \mathrm{mmol} / 1$ na primeira para $158,3 \pm 5,8 \mathrm{mmol} / 1$ na quarta consulta em 13 cães testados.

\section{DISCUSSÃO}

Os inibidores da ECA são usados no tratamento da ICC porque previnem o remodelamento ventricular, causam regressão da hipertrofia ventricular esquerda e reduzem a pré e pós-carga, suprimindo sistêmica e localmente o SRA. O uso de enalapril aumenta a expectativa de vida de cães com ICC, especialmente decorrente de cardiomiopatia dilatada (Controlled..., 1995). O benazepril, em combinação ou não com terapia cardíaca convencional, produz melhoras clínicas (Pouchelon et al., 1996; Kitagawa et al., 1997) e aumenta a expectativa de vida (Pouchelon, 1999) de cães com insuficiência cardíaca. Nos pacientes do presente estudo, a melhora clínica foi caracterizada por diminuição da dispnéia, da freqüência da tosse, do número de síncopes e normalização da coloração das mucosas. Houve resolução do edema pulmonar e diminuição dos sinais radiográficos de congestão pulmonar. No Improve Study Group (Acute..., 1995), a melhora aguda foi observada em cães que receberam enalapril em adição à terapia padrão, havendo diminuição da pressão arterial, da pressão capilar pulmonar sem alteração no débito cardíaco. Embora essas melhoras agudas não sejam intensas, são, em parte, responsáveis pela diminuição da congestão e do edema pulmonar observados. Como foram utilizados outros fármacos que podem ter auxiliado na diminuição do volume circulante (especialmente diuréticos), não é possível atribuir apenas ao benazepril toda melhora dos sinais clínicos observados no presente estudo.

Dos casos da presente pesquisa, três dos 11 cães com CMD e um dos nove com endocardiose de mitral morreram de ICC, diferentemente dos resultados obtidos em um estudo prospectivo (Controlled..., 1995), em que, dos 211 cães com 
ICC que haviam recebido enalapril ou placebo, avaliados durante 28 dias, sete que receberam placebo morreram. As diferenças na mortalidade provavelmente não são decorrência de diferenças entre o enalapril e o benazepril, porque esses fármacos têm propriedades farmacocinéticas e efeitos similares em cães (Hamlin e Nakayama, 1998). Além disso, o uso de benazepril aumenta a expectativa de vida de cães com ICC, em relação a cães que recebem placebo (Pouchelon, 1999). A maior mortalidade no presente estudo é provavelmente resultado do maior tempo de observação (56 dias versus 28 dias) e da severidade da insuficiência cardíaca. A expectativa de vida de cães com cardiomiopatia dilatada que recebem diuréticos e digoxina sem um inibidor da ECA é de apenas 58 dias, chegando a 157 dias em cães que recebem enalapril (Controlled..., 1995). Neste estudo, dois cães com ICC severa tinham menos de um ano de idade. Em Dobermans com menos de dois anos de idade, a cardiomiopatia dilatada progride mais rápido do que em cães mais velhos (Sisson et al., 1999). Conseqüentemente, a morte de apenas três de 11 cães com miocardiopatia dilatada, dos quais dois tinham menos de um ano de idade, em um período de 56 dias, não é um achado inesperado e sim conseqüência do provável agravamento da cardiopatia pela desautonomia parassimpática com ativação simpática, decorrente da degeneração dos neurônios ganglionares atriais que acometem cães com cardiomiopatia dilatada (Camacho, 1996). É importante ressaltar que o presente estudo não foi duplo-cego e não houve a utilização de placebo, o que difere dos estudos anteriores (Controlled..., 1995; Pouchelon, 1999).

A melhora da dispnéia é em parte decorrente da diminuição do edema pulmonar visualizado radiograficamente. Em alguns pacientes com ICC moderada, no entanto, a melhora da dispnéia não pode ser correlacionada com a melhora radiográfica. É possível que, nesses casos, a diminuição da pós-carga tenha aumentado o débito cardíaco e diminuído a ativação dos ergorreceptores musculares. Os ergorreceptores musculares participam na mediação da dispnéia e na sensação de fadiga muscular em pacientes com ICC (De Morais, 2000). A diminuição da atividade dos ergorreceptores é associada à diminuição da dispnéia e ao aumento da tolerância ao exercício. Embora apenas a redução da dispnéia tenha diminuído no presente estudo, o número de cães apresentando intolerância ao exercício diminuiu de 11 para nove. É interessante notar que, em cães com ICC experimental devido à regurgitação de valva mitral, o aumento na tolerância ao exercício não é um efeito imediato. Até os 19 dias após o início da terapia, cães que receberam enalapril apresentavam melhor desempenho do que o grupo placebo (Hamlin et al., 1996), sugerindo que esse efeito não se deve exclusivamente à diminuição do edema pulmonar. $\mathrm{O}$ mecanismo pelo qual os inibidores da ECA melhoram a tolerância ao exercício envolve a redução da impedância aórtica, a redução da resistência arterial sistêmica e a alteração na distribuição do débito cardíaco para os músculos locomotores, com diminuição da atividade dos ergorreceptores (Hamlin et al., 1996).

No presente estudo, não foi encontrada nenhuma alteração laboratorial clinicamente importante, o que evidencia que o benazepril, assim como o enalapril (Controlled..., 1995), não comprometeu a função renal. A concentração sérica de creatinina aumentou em um grupo de cães que recebeu benazepril, mas esse aumento ficou dentro dos valores de normalidade (Kitagawa et al., 1997). Em um estudo prospectivo duplo-cego de longa duração em cães com ICC tratados com benazepril, observou-se menor possibilidade de apresentar elevações da creatinina que o grupo que recebeu placebo (Pouchelon, 1999). Não ocorreram alterações na concentração de potássio, a exemplo do que já foi observado anteriormente com o uso de benazepril e enalapril (Controlled..., 1995; Pouchelon, 1999). O uso concomitante de diuréticos na maioria dos pacientes avaliados nesses estudos pode ter contrabalançado a tendência dos inibidores da ECA de reter potássio. Além disso, os inibidores da ECA só costumam causar hipercalemia em cães que recebem suplementação de potássio ou com comprometimento da função renal (Dibartola e De Morais, 2000). Houve um aumento significativo na concentração sérica de sódio após o uso de benazepril no presente estudo. A concentração de sódio reflete a quantidade de água livre existente no organismo. Em pacientes com ICC, a ativação do SRA leva ao aumento da sede e da concentração de ADH, podendo, em casos mais severos, ocorrer hiponatremia (De Morais, 2000). É possível que o bloqueio do SRA pelo benazepril tenha 
causado diminuição na sede e na concentração de $\mathrm{ADH}$, ocasionando diminuição da água livre e aumento da concentração sérica de sódio. Embora não possa se excluir que a furosemida tenha contribuído para o aumento do sódio, normalmente esse diurético causa mais natriurese do que diurese aquosa, o que tenderia a causar hiponatremia (Bonagura et al., 2000).

Quanto a alterações de ritmo cardíaco, apenas um cão apresentou taquicardia ventricular. Em pacientes humanos com ICC, os inibidores da ECA têm efeitos antiarrítmicos. Entre os fatores que poderiam contribuir para esse efeito antiarrítmico, incluem-se: aumento do potássio sérico, redução da sobrecarga e distensão ventricular, redução do consumo de oxigênio e da isquemia miocárdica, atenuação da atividade simpática e dos níveis circulantes de noradrenalina (Batlouni, 1991). Embora os inibidores da ECA tenham efeito vasodilatador, não foi observado nenhum caso de hipotensão arterial no presente estudo. A diminuição da pressão arterial decorrente do uso do enalapril em cães com ICC é baixa, o que explica por que a ocorrência de hipotensão é incomum em cães com ICC que recebem enalapril ou benazepril (Acute..., 1995; Kitagawa et al., 1997).

Neste estudo foi possível observar que o benazepril, associado ou não a diuréticos e digitálicos, é um agente efetivo e seguro no tratamento da ICC severa e moderada em cães, concondando com estudos feitos anteriormente (Controlled..., 1995; Pouchelon, 1996; Pouchelon, 1999).

\section{AGRADECIMENTOS}

À FAPESP e à empresa Novartis Saúde Animal Ltda. pelo apoio financeiro no desenvolvimento da pesquisa.

\section{REFERÊNCIAS BIBLIOGRÁFICAS}

ACUTE and short-term hemodynamic, echocardiographic, and clinical effects of enalapril maleate in dogs with naturally acquired heart failure: results of the invasive multicenter prospectve veterinary evaluation of enalapril study. The improve study group. J. Vet. Int. Med., v.9, p.234-242, 1995.

AWAN, N.A.; MASON, D.T. Direct seletive blockade of the vascular angiotensin II receptors in therapy for hypertension and severe congestive heart failure. Am. Heart J., v.131, p.177-185, 1996.

BATLOUNI, M. Insuficiência cardíaca: da fisiologia ao tratamento. Parte I: fisiologia. Arq. Bras. Cardiol., v.57, p.63-73, 1991.

BONAGURA, J.D.; LEHMKUHL, L.B.; DE MORAIS, H.S.A. Fluid and diuretic therapy in heart failure. In: DIBARTOLA, S.P. Fluid therapy in small animal practice. 2.ed. Philadelphia: W.B. Saunders, 2000. p.387-427.

BUCHANAN, J.W. Prevalence of cardiovascular disorders. In: FOX, P.R.; SISSON, D.; MOÏSE, N.S. Textbook of canine and feline cardiology: principles and clinical practice. 2.ed. Philadelphia: W. B. Saunders, 1999. p.457-470.

CALVERT, C.A. Effect of medical therapy on survival of patients with dilated cardiomyopathy. Vet. Clin. North Am.: Small. Anim. Pract., v.21, p.919-930, 1991.

CAMACHO, A.A. Estudo clínico, caracterização morfométrica e quantitativa dos neurônios ganglionares atriais da faixa intercaval de cães com miocardiopatia dilatada congestiva idiopática. 1996. 56f. Tese (Livre Docência) - Faculdade de Ciências Agrárias e Veterinárias, Universidade Estadual Paulista, SP.

CONTROLLED clinical evaluation of enalapril in dogs with heart failure: results of the Cooperative Veterinary Enalapril Study Group. J. Vet. Int. Med., v.9, p.243-252, 1995.

DE MORAIS, H. A. Pathophysiology of heart failure and clinical evaluation of cardiac function. In: ETTINGER, S.J., FELDMAN, E.C. Textbook of veterinary internal medicine. 5.ed. Philadelphia: W. B. Saunders, 2000. p.692-712.

DIBARTOLA, S.P.; DE MORAIS, H.S.A. Disorders of potassium: Hypokalemia and hyperkalemia. In: DIBARTOLA, S.P. Fluid therapy in small animal practice. 2.ed. Philadelphia: W.B. Saunders, 2000. p.83-107.

ETTINGER, S.J.; BENITZ, A.M.; ERICSSON, G.F. et al. Effects of enalapril maleate on survival of dogs with naturally acquired heart failure. The long-term investigation of veterinary 
enalapril (live) study group. J. Am. Vet. Med. Assoc., v.213, p.1573-1577, 1998.

HAMLIN, R.L.; BENITZ, A.M.; ERICSSON, G.F. et al. Effects of enalapril on exercise tolerance and longevity in dogs with heart failure produced by iatrogenic mitral regurgitation. $J$. Vet. Int. Med., v.10, p.85-87, 1996.

HAMLIN, R.L.; NAKAYAMA, T. Comparison of some pharmacokinetic parameters of 5 angiotensin-converting enzyme inhibitors in normal beagles. J. Vet. Int. Med., v.12, p.93-95, 1998.

INSEL, J.; MIRVIS, D.M.; BOLAND, M.J. et al. A multicenter study of the safety and efficacy of benazepril hydrochloride, a long-acting angiotensin-converting enzyme inhibitor, in patients with chronic congestive heart failure. Clin. Pharmacol. Ther., v.45, p.312-320, 1989.

KATZ, A M. The cardiomyopathy of overload: na unnatural growth response in the hypertrophied heart. Ann. Int. Med., v.121, p.363-371, 1994.

KING, J.N.; MAURON, C.; KAISER, G. Pharmacokinetics of the active metabolite of benazepril, benazeprilat, and inhibition of plasma angiotensin-converting enzyme activity after single and repeated administrations to dogs. Am. J. Vet. Res., v.56, p.1620-1628, 1995.

KITAGAWA, H.; EGUCHI, T.; KITOH, K. et al. Plasma concentrations of an angiotensinconverting enzyme inhibitor, benazepril, and its active metabolite, benazeprilat, after repeated administrations of benazepril in dogs with experimental kidney impairment. J. Vet. Med. Sci., v.62, p.179-185, 2000.

KITAGAWA, H.; WAKAMIYA, H.; KITOH, $K$. et al. Efficacy of monotherapy with benazepril, na angiotensin converting enzyme inhibitor, in dogs with naturally acquired chronoc mitral insufficiency. J. Vet. Med. Sci., v.59, p.513-520, 1997.

KNIGHT, D.H. Fisiopatologia da insuficiência cardíaca. In: In: ETTINGER, S.J. Tratado de medicina interna veterinária. São Paulo: Manole, 1992. p.944-969.
LORD, P.F.; SUTER, P.F. Radiology. In: FOX, P.R.; SISSON, D.D.; MOÏSE, N.S. Textbook of canine and feline cardiology. 2.ed. Philadelphia: W.B. Saunders, 1999. p.107-129.

PONIKOWSKI, P.; CHUA,T.P.; PIEPOLI, M. Ventilatory response to exercise correlates with imparied heart rate variability in patients with chronic congestive heart failure. Am. J. Cardiol., v.82, p.338-344, 1998.

POUCHELON, J.L. The effect of benazepril on survival times and clinical signs of dogs with congestive heart failure: results of a multicenter, prospective, randomized, double-blinded, placebo-controlled, long-term clinical trial. $J$. Vet.Cardiol., v.1, p.7-18, 1999.

POUCHELON, J.L. Treatment of heart failure in dogs with benazepril - results of European double-blind placebo controlled study. In: AMERICAN COLLEGE OF VETERINARY INTERNAL MEDICINE FORUM, 14., 1996. Proceedings... 1996. p.745.

ROTH, A.L. Use of angiotensin-convertinginhibitors in dogs with congestive heart failure. Compend. Cont. Educ., v.15, p.1240-1244, 1993.

SCHLESINGER, D.P.; RUBIN, S.I. Potential adverse effects of angiotensin-converting enzyme inhibitors in the treatment of congestive heart failure. Compend. Cont. Educ., v.16, p.275283, 1994.

SISSON, D. Evidence for or against the efficacy of afterload reducers for management of heart failure in dogs. Vet. Clin. North Am.: Small Anim. Pract., v.21, p.945-955, 1991.

SISSON, D.D.; O'GRADY, M.R.; CALVERT, C.A. Myocardial disease in dogs. In: FOX, P.R.; SISSON, D.; MOÏSE, N.S. Textbook of canine and feline cardiology: Principles and clinical practice. 2.ed. Philadelphia: W.B. Saunders, 1999. p.581-619.

SNYDER, P.S. Canine hipertensive disease. Compend. Cont. Educ., v.13, p.1785-1792, 1991.

WEBER, K.T. Heart failure: lessons learned over the past 25 years. Clin. Cardiol., v.18, p.23-130, 1995. 